\title{
Esthetic restoration of severely stained fluorosed teeth: a case report
}

\section{Introduction}

An individual appearance and his or her perception of it has a major impact on his or her self-esteem; reflects a person's overall subjective emotional evaluation of their worth. ${ }^{1,2}$ That's said, appearance and its perception affect the person quality of living and social interaction. ${ }^{3,4}$ The smile is the first thing people notice when meeting other people for the first time. Discolored teeth affect smile majorly according to the severity of discoloration, were minor discoloration can be unnoticeable, but as it becomes darker, it becomes more noticeable and thus the more dramatic effect of the patient smile awareness; Besides the loss of the Vertical Dimension of Occlusion affect esthetics and function, a person will appear older with dropped mouth corner and raised chin ${ }^{5,6}$ as well as adversely affecting the mastication process and supporting tissue health..$^{5-7}$ The case discussed in this article was self-conscious as explained by him due to his darkened teeth as well chewing difficulties, it's quite noticeable the impact of teeth esthetic alteration on his self-esteem at the end of treatment.

\section{Clinical report}

A 22 Yemeni male patient presented to the College of Dentistry clinics at the University of Dammam seeking cosmetic treatment with a chief complaint "I want to improve the way my teeth look " $\mathrm{He}$ reported no significant medical history. Patient has never been into the dental clinic. He stated his deciduas teeth were normal and his whole village complains of darkened teeth (brownish). A panoramic $\mathrm{x}$-ray (Figure 1), intraoral pictures (Figures 2-6) as well as study casts were taken to diagnose the case (Figure 7). Upon examination, the patient was found to suffer from severely generalized discoloration of permanent teeth and moderate dental attrition resulting in shortening of the vertical dimension of occlusion (VDO). A class I occlusion with class I canine relation was present, with an over-bite of $4 \mathrm{~mm}$ and an overjet of $4 \mathrm{~mm}$. The upper lip seems stretched with dropping mouth angles. The patient suffers from generalized marginal chronic gingivitis with no mobility or furcation involvement noticed. The treatment plan started with plaque control measures were conducted as well as patient education. The patient's esthetic problem caused by Fluorosis both teeth staining and facial appearance and occlusion. The treatment options available range from bleaching, porcelain veneers, full coverage ceramic crowns or porcelain fused to metal crowns. Due to the Fluorosis severity, the bond strength of the adhesive agent was questionable that eliminated the veneers option. Bleaching when tried had minimal effects. Finally, porcelain-fused to metal (PFM) crowns were selected to restore esthetics of anterior teeth, while posterior gold crowns were chosen to raise the occlusion posterior by $2 \mathrm{~mm}$. Treatment options were discussed with the patient who consents to the treatment plan.

Study casts were poured and mounted according to registered intra occlusal records on which an occlusal splint was made first with a thickness of $1 \mathrm{~mm}$; the patient was instructed to wear it for 3 weeks. When the splint thickness was increased into $2 \mathrm{~mm}$, the patient complained after 1 week wearing the splint. Accordingly, the thickness of the splint was reduced to $1.5 \mathrm{~mm}$. After wearing the splint
Volume 10 Issue 2 - 2019

\author{
Rasha AISheikh,' Muntasar T Al Hinai ${ }^{2}$ \\ 'Assistant Professor, Department of Restorative Dental \\ Sciences, College of Dentistry, Imam Abdulrahman Bin Faisal \\ University, Saudi Arabia \\ ${ }^{2}$ Consultant in Orthodontics and Dentofacial Orthopedics, \\ Sultan Qaboos University Hospital, Muscat, Sultanate of Oman

\begin{abstract}
Correspondence: Rasha Numan AISheikh, Department of Restorative Dental Science, College of Dentistry, Imam Abdulrahman Bin Faisal University, P.O.Box 30018,Alkhobar 31952, Saudi Arabia, Tel +966 I3 333 I452, +966 5068648|4, Email ralsheikh@iau.edu.sa, ralsheik@gmail.com
\end{abstract}

Received: March 06, 2019 | Published: March 12, 2019

for two more weeks with no complaints, the splint was then used in the lab to help to construct temporary crowns; the procedure included dividing the splint at the midline to help to mount the cast with the required VDO. One side of the splint was left in place to be used as a guide for the required VD while constructing temporary crowns for the other hand. At the clinic posterior teeth were prepared; first, temporary crowns were constructed following the wax up made in the lab to raise the VDO. The anterior teeth were then prepared and temporary crowns were placed (Figure 8 ). The patient complained of pain in the lower right premolar area, upon examination tooth \# 28 needed root canal treatment.

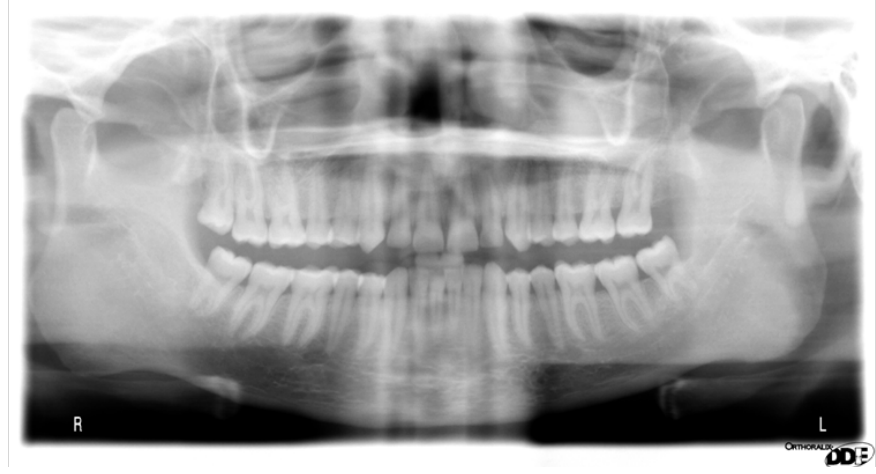

Figure I Pre op OPG.

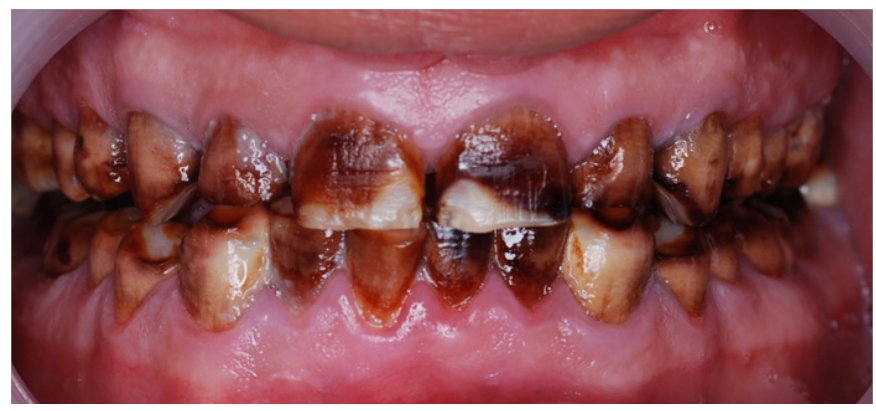

Figure 2 Pre op front view. 


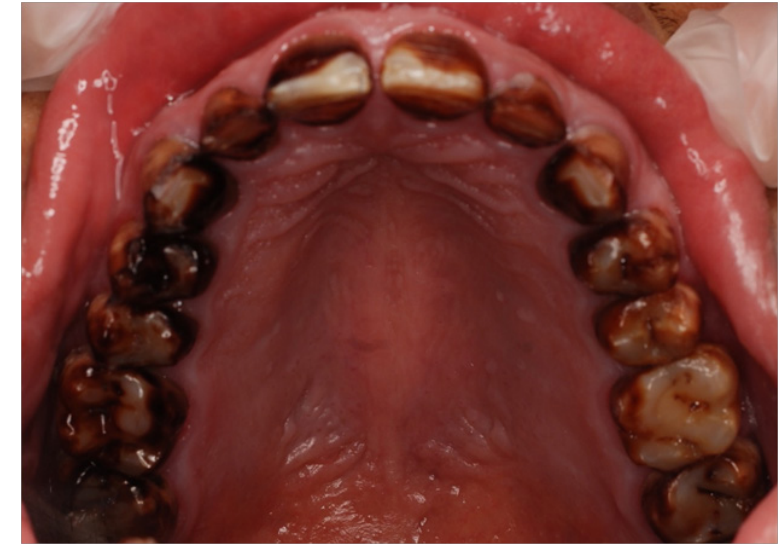

Figure 3 Pre op upper arch.

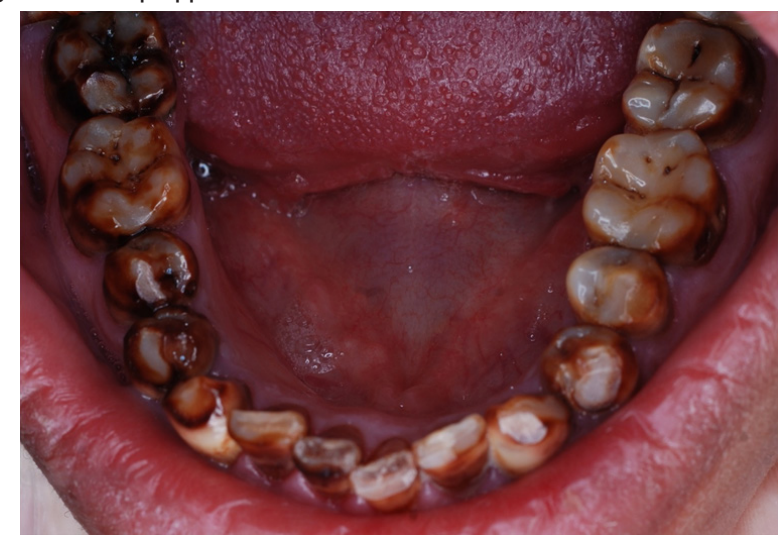

Figure 4 Pre op lower arch.

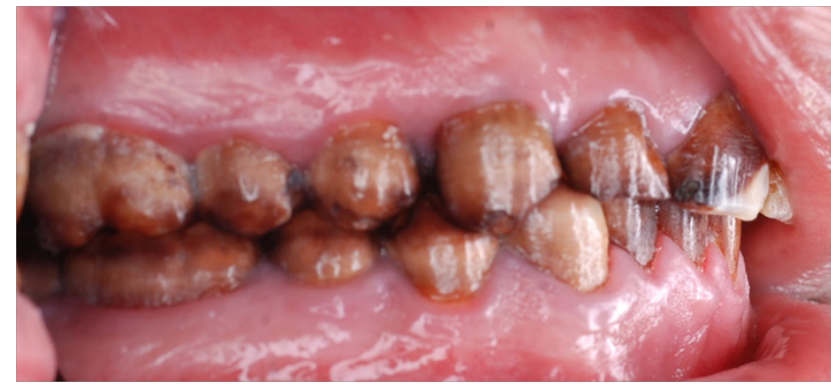

Figure $\mathbf{5}$ Pre op left side.

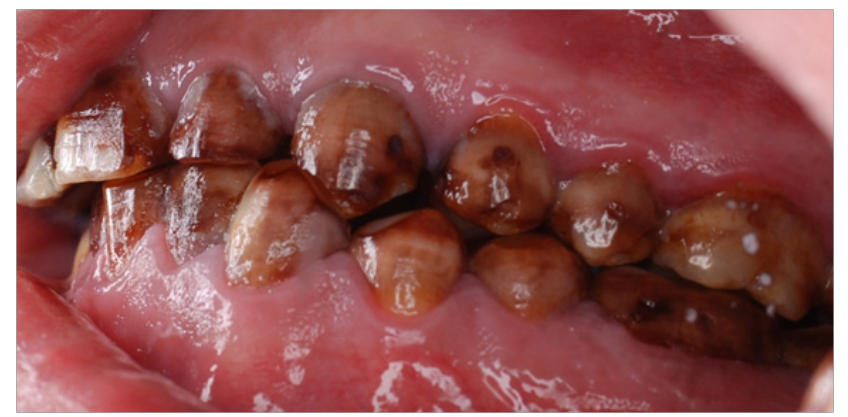

Figure 6 Pre op right side.

Esthetic, phonetics and occlusion were checked after cementation the temporary crowns with temporary (Pro. Temp) luting agent. To avoid any functional problems, the patient was instructed to give the temporaries a period of trial for 2 weeks. Intra occlusal relationship was repeated at this stage again. When the patient reported no discomfort, the final crowns were constructed. Final crowns were cemented using resin composite (Rely X Unicem) luting agent. Teeth restored were \# 2, 3, 4, 5, 6, 7, 8, 9,10, 11, 12, 13, 14, 15,18, 19, 20, $21,22,23,24,25,26,27,28,29,30$ and 31. Postoperative pictures, $\mathrm{x}$-rays, and impressions were taken (Figure 9-19). The case had a good prognosis considering the patient, good general health and proper attitude toward his oral hinging reported during the maintenance and treatment phases. The periodontium is in functional health status, using gold alloys to restore posterior teeth as well as porcelain-fused to metal crowns to restore anterior teeth conserve tooth structures by minimizing the amount of tooth structure to be cut.

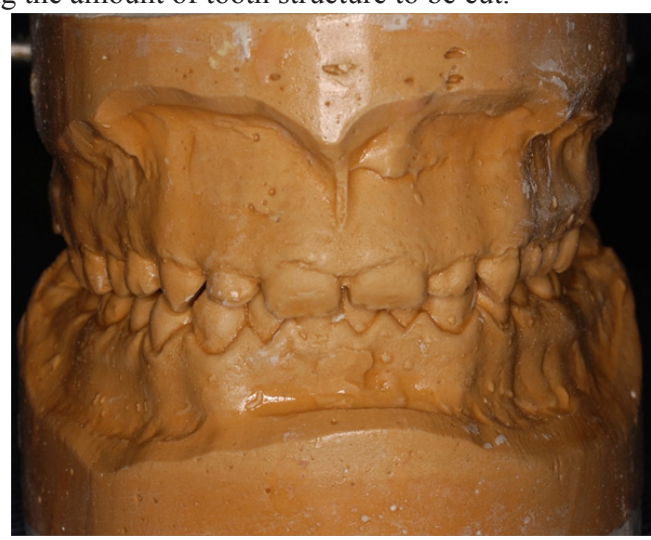

Figure 7 Study cast front.

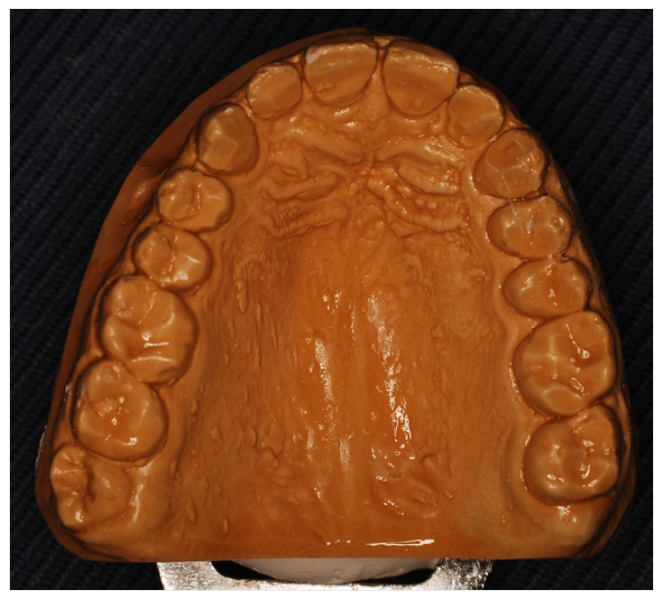

Figure 8 Study cast upper.

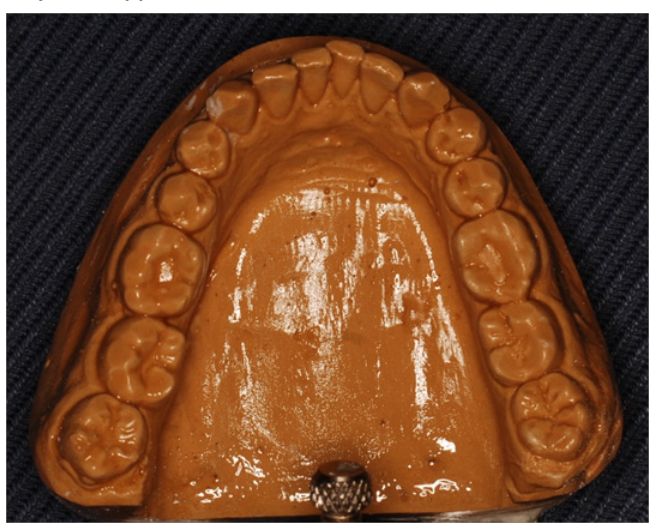

Figure 9 Study cast lower. 


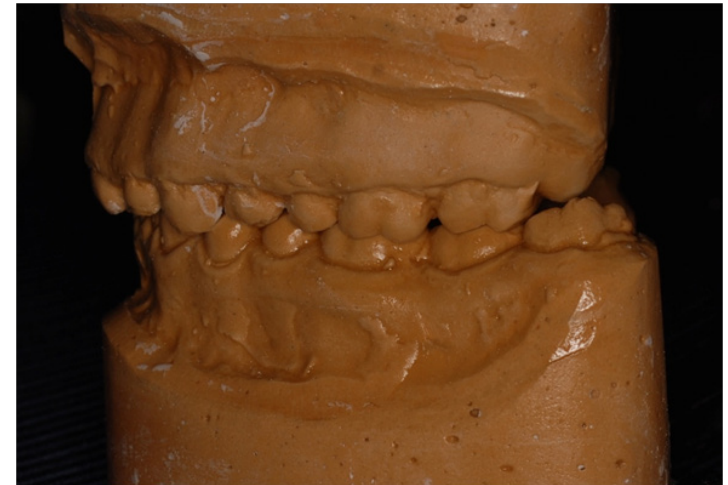

Figure 10 Study cast left.

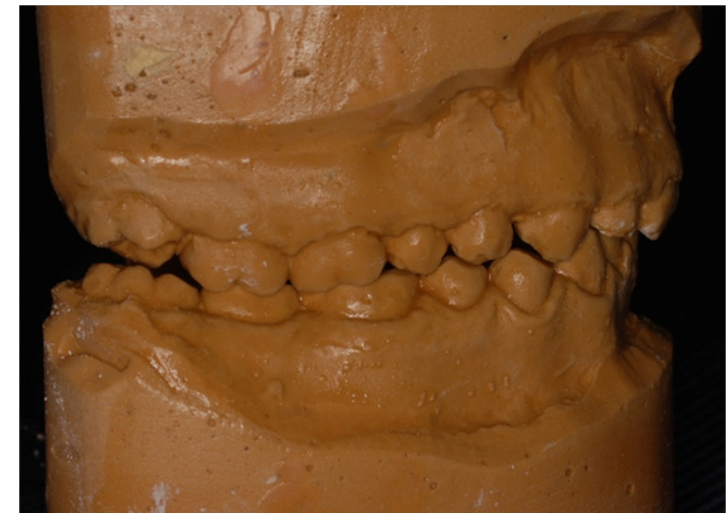

Figure I I Study cast right.

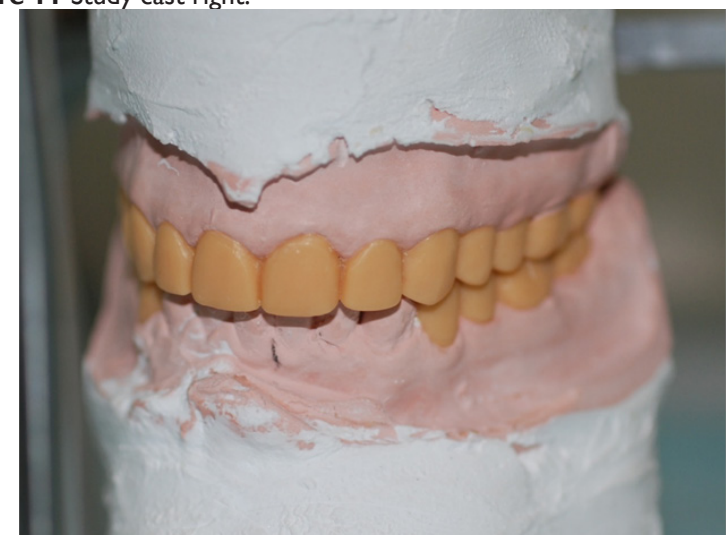

Figure 12 Study wax-up.

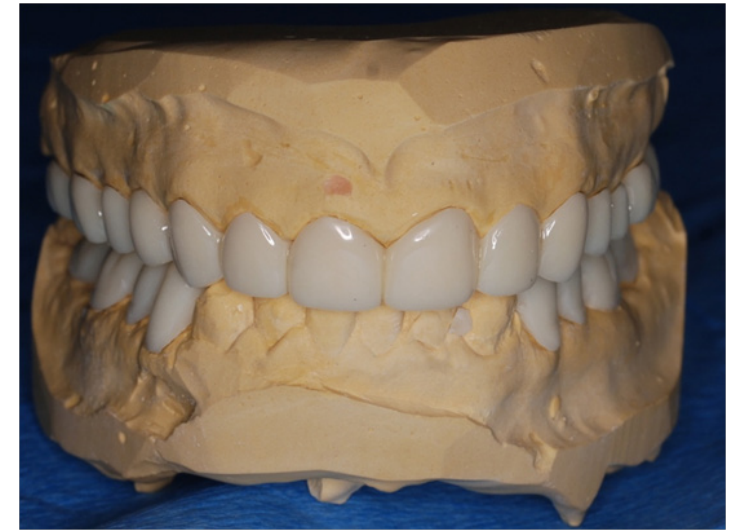

Figure 13 Temporrays.

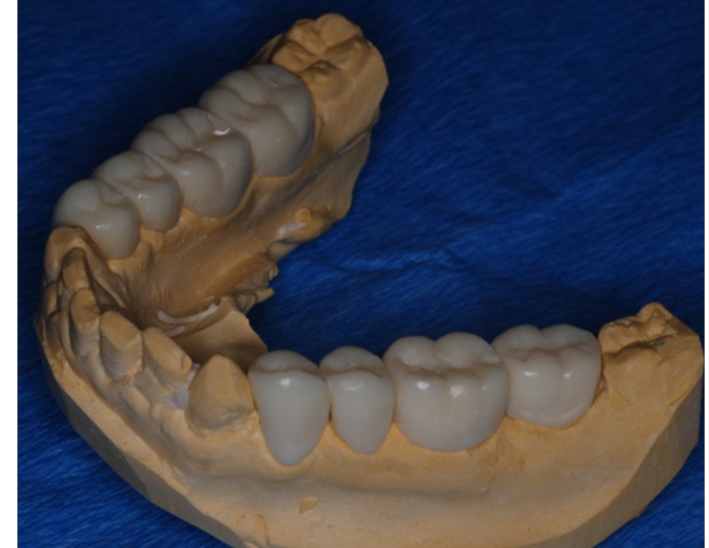

\section{Figure 14 Temporarys 2.}

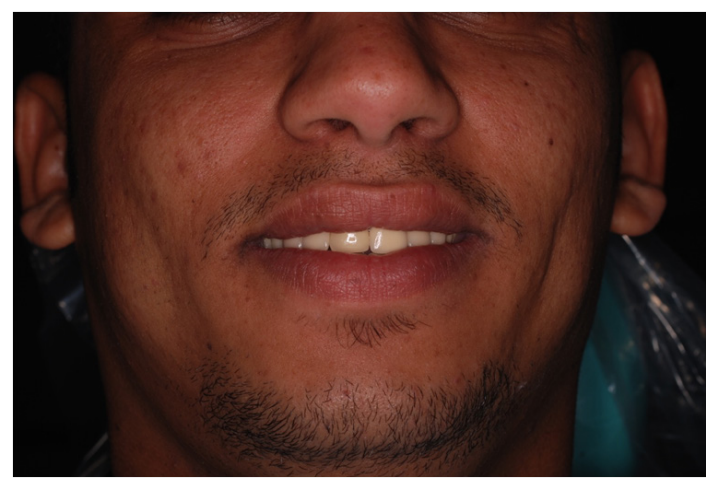

Figure I5 Final frontal view.

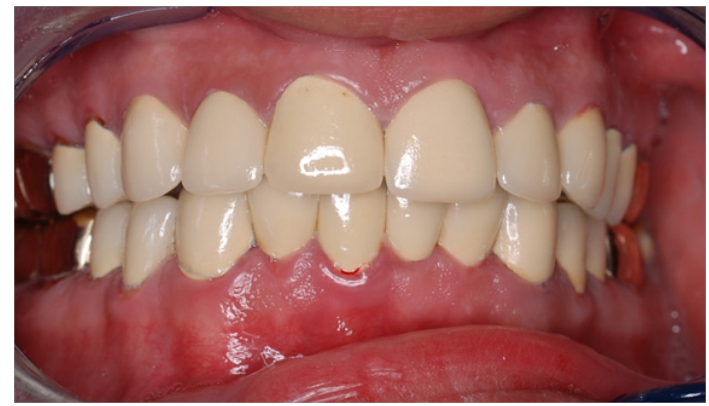

Figure 16 Final front view.

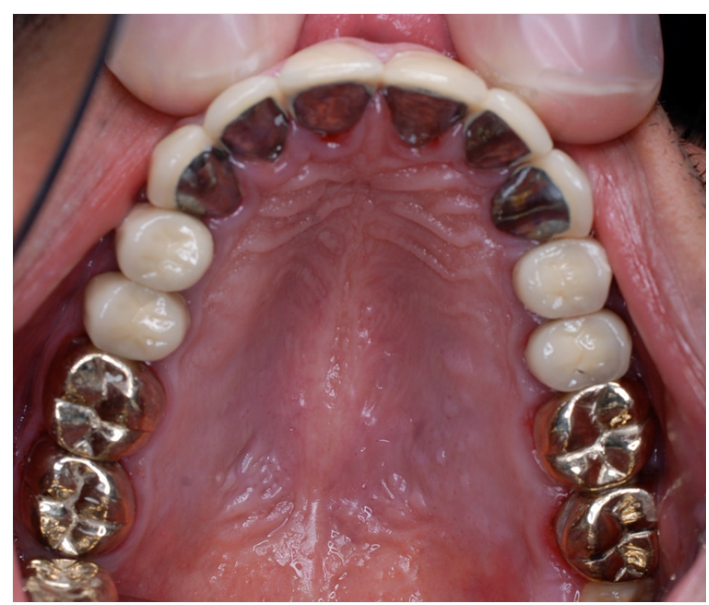

Figure 17 Final upper arch view.

Citation: AlSheikh R, Hinai MTA. Esthetic restoration of severely stained fluorosed teeth: a case report.J Dent Health Oral Disord Ther. 2019;10(2): I 12-II6. DOI: 10.15406/jdhodt.2019.10.00470 


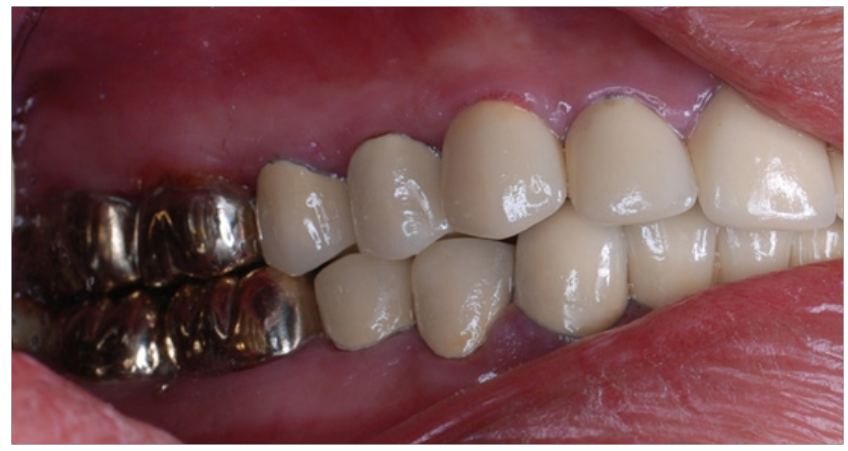

Figure 18 Post op left side.

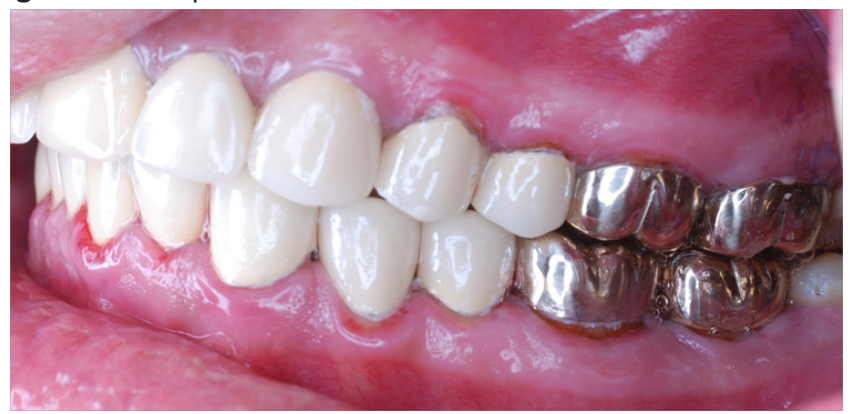

Figure 19 Final right view.

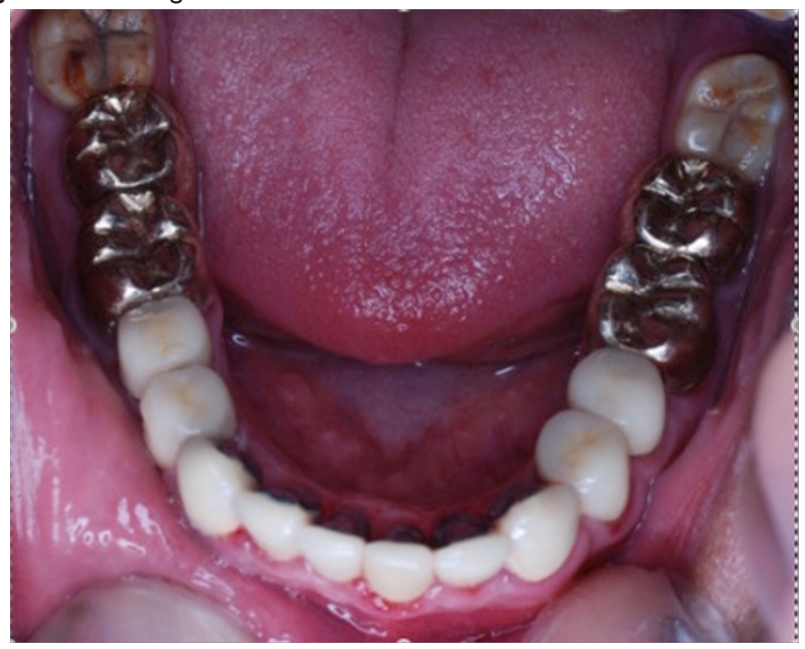

Figure 20 Final lower arch view.

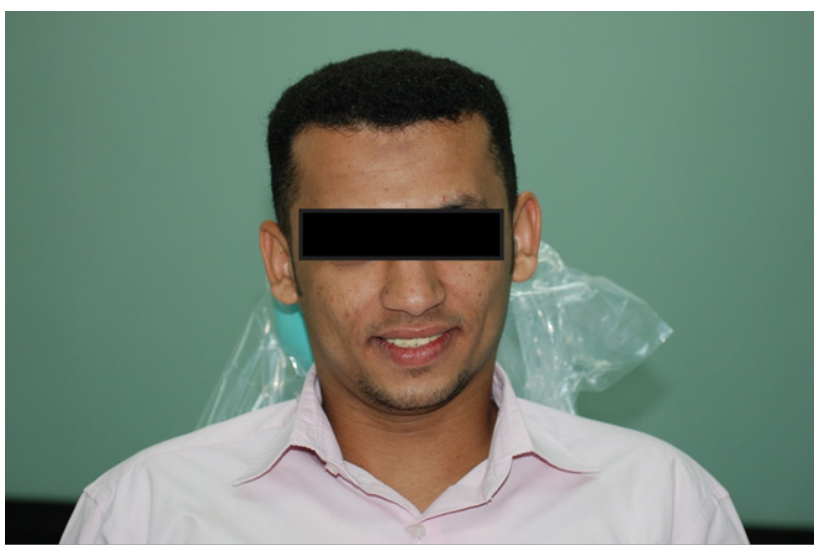

Figure 2I Post op facial view.

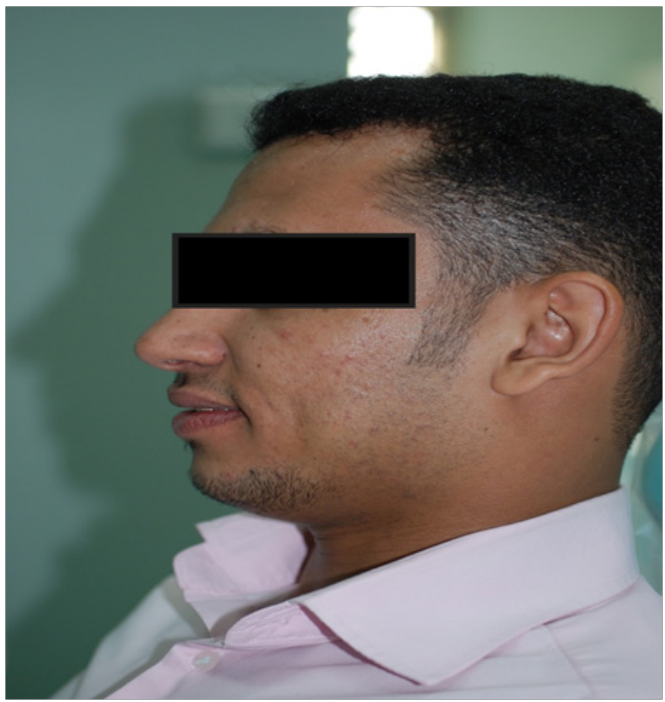

Figure 22 Post op profile.

\section{Discussion}

Fluoridated water is considered one of the most critical public health improvement measures ${ }^{8}$ in caries prevention; giving the amount of fluoride in the water is carefully calculated. A small amount of fluoride found to be effective (up to 1ppm Fluoride), amounts above 2ppm will lead to dental Fluorosis. ${ }^{49-11}$ Yemen; due to political situation drinking water is treated to be suitable for drinking and cooking; no control is paid to the mineral content none the less Fluoride content. Giving most of the drinking water came from natural resources namely springs and underground waters fluoride content found to be higher than the recommended percent. ${ }^{12,13}$ Rahimah AK et. Al. published a paper in 2010 stating that $30 \%$ of the population of Yemen suffer from varying degree of dental Fluorosis the percent is higher in male compared to females. ${ }^{13}$ When the patient walked into the clinic the differential diagnosis of our clinical findings was Amelogenesis Imperfecta, which affect both deciduas and permanent dentition, ${ }^{14}$ patients stated his deciduous teeth were normal. Environmental Enamel Hypoplasia was another diagnosis it can be caused by a nutritional deficiency which is usually localized affecting both enamel and dentin to some extent, ${ }^{15}$ Congenital syphilis which affects a localized area usually the centrals and first molars, ${ }^{16}$ Birth energies also affect localized areas and last Fluorosis. Giving the patient ethnic background, dental history and clinical condition the final diagnosis found to be dark dental discoloration caused by severe dental Fluorosis. In mild to moderate Fluorosis esthetics is not a significant concern as the alteration in tooth appearance is minimal and the color change can be reversed with vital teeth bleaching, in the other hand sever case patients suffer from dark teeth discoloration (dark brown) as the defected enamel continue to uptake and retain pigmintation. ${ }^{17-21}$ Dental Fluorosis is a tooth malformation caused by chronic digestion of fluoride resulting in a hypo mineralized enamel, ${ }^{22}$ characterized by a thin high-mineralized outer layer resistance to acid,,$^{23}$ and an inner porous enamel characterized by high permeability. ${ }^{24}$ bonding to Fluorosed enamel has always been a challenge, enamel with high acid resistance at the same time the resultant bond strength is lower than natural teeth. ${ }^{25,26}$ Taking this in consideration and the fact that the vertical dimension of occlusion has to be raised porcelain fused to metal crown were selected to restore function and esthetic 
of anterior teeth and ensure conservatism of tooth structure as well as longevity of the restoration. A similar approach was used in other similar cases. ${ }^{27}$

\section{Summary}

Treatment modalities if needed to restore esthetic of fluorosed teeth varies according to patients need, other existing conditions an important selecting factor rely on conservatism as well as longevity and patient satisfaction. For this case, a full mouth rehabilitation was found to me most suitable, done through raising the vertical dimension using full coverage gold crowns for posterior teeth and porcelain fused to metal crowns for anterior teeth such restoring form, esthetic and function.

\section{Acknowledgments}

None.

\section{Conflicts of interest}

The author declares that there is no conflict of interest.

\section{References}

1. Hewitt JP. Oxford Handbook of Positive Psychology. oxford: Oxford University Press; 2009.

2. Almanea R, Modimigh A, Almogren F, et al. Perception of smile attractiveness among orthodontists, restorative dentists, and laypersons in Saudi Arabia. J Conserv Dent. 2019;22(1):69-75.

3. Klages U, Erbe C, Sandru SD, et al. Psychosocial impact of dental aesthetics in adolescence: validity and reliability of a questionnaire across age-groups. Qual Life Res. 2015;24(2):379-390.

4. Nomura S, Freitas KMS, Silva P, et al. Evaluation of the attractiveness of different gingival zeniths in smile esthetics. Dental Press J Orthod. 2018;23(5):47-57.

5. Liu Z, McGrath C, Hagg U. The impact of malocclusion/orthodontic treatment need on the quality of life. A systematic review. Angle Orthod. 2009;79(3):585-591.

6. Badran SA. The effect of malocclusion and self-perceived aesthetics on the self-esteem of a sample of Jordanian adolescents. Eur J Orthod. 2010;32(6):638-644.

7. Burke FJ. Information for patients undergoing treatment for toothwear with resin composite restorations placed at an increased occlusal vertical dimension. Dent Update. 2014;41(1):28-38.

8. Recommendations for using fluoride to prevent and control dental caries in the United States. Centers for Disease Control and Prevention. MMWR Recomm Rep. 2001;50(RR-14):1-42.

9. Lennon MA. One in a million: the first community trial of water fluoridation. Bull World Health Organ. 2006;84(9):759-760.

10. Ranasinghe N, Kruger E, Chandrajith R, et al. The heterogeneous nature of water well fluoride levels in Sri Lanka: An opportunity to mitigate the dental fluorosis. Community Dent Oral Epidemiol. 2019.
11. Aghapour S, Bina B, Tarrahi MJ, et al. Distribution and health risk assessment of natural fluoride of drinking groundwater resources of Isfahan, Iran, using GIS. Environ Monit Assess. 2018;190(3):137.

12. Akpata ES, Fakiha Z, Khan N. Dental fluorosis in 12-15-year-old rural children exposed to fluorides from well drinking water in the Hail region of Saudi Arabia. Community Dent Oral Epidemiol. 1997;25(4):324-327.

13. Kadir RA,Al-Maqtari RA. Endemic fluorosis among 14-year-old Yemeni adolescents: an exploratory survey. Int Dent J. 2010;60(6):407-410.

14. Raine J, Winter RM, Davey A, Tucker SM. Unknown syndrome: microcephaly, hypoplastic nose, exophthalmos, gum hyperplasia, cleft palate, low set ears, and osteosclerosis. J Med Genet. 1989;26(12):786-788.

15. Hillmann G, Geurtsen W. Pathohistology of undecalcified primary teeth in vitamin D-resistant rickets: review and report of two cases. Oral Surg Oral Med Oral Pathol Oral Radiol Endod. 1996;82(2):218-224.

16. Khetarpal S, Kempf E, Mostow E. Congenital syphilis: early- and latestage findings of rhagades and dental anomalies. Pediatr Dermatol. 2011;28(4):401-403.

17. Zavala-Alonso V, Martinez-Castanon GA, Patino-Marin N, et al. Characterization of healthy and fluorotic enamel by atomic force microscopy. Microsc Microanal. 2010;16(5):531-536.

18. Levy SM. An update on fluorides and fluorosis. J Can Dent Assoc. 2003;69(5):286-291.

19. McKnight CB, Levy SM, Cooper SE, et al. A pilot study of esthetic perceptions of dental fluorosis vs. selected other dental conditions. ASDC J Dent Child. 1998;65(4):233-229.

20. Riordan PJ. Perceptions of dental fluorosis. $J$ Dent Res. 1993;72(9):1268-1274.

21. Chankanka O, Levy SM, Warren JJ, et al. A literature review of aesthetic perceptions of dental fluorosis and relationships with psychosocial aspects/oral health-related quality of life. Community Dent Oral Epidemiol. 2010;38(2):97-109.

22. Vieira AP, Hancock R, Limeback H, et al. Is fluoride concentration in dentin and enamel a good indicator of dental fluorosis? $J$ Dent Res. 2004;83(1):76-80.

23. Silva-Benitez EL, Zavala-Alonso V, Martinez-Castanon GA, et al. Shear bond strength evaluation of bonded molar tubes on fluorotic molars. Angle Orthod. 2013;83(1):152-157.

24. Newburn E, Brudevold F. Studies on the physical properties of fluorosed enamel. I. Microradiographic studies. Arch Oral Biol. 1960;2:15-20.

25. Miller RA. Bonding fluorosed teeth: new materials for old problems. $J$ Clin Orthod. 1995;29(7):424-427.

26. Adanir N, Turkkahraman H, Yalcin Gungor A. Effects of adhesion promoters on the shear bond strengths of orthodontic brackets to fluorosed enamel. Eur J Orthod. 2009;31(3):276-280.

27. Rajesh P, Prasad M, Haldal S. Full mouth rehabilitation of a patient with amelogenesis imperfecta: a case report. $J$ Int Oral Health. 2014;6(4):76-79. 